\title{
0 Uso das Bases de Dados Espectrais da Bio-Rad Informatics Division em Correlações Espectro-Estrutura de Polímeros
}

\author{
A Revista apresenta nesta edição uma matéria que foi elaborada pelo Prof. Dr. João Carlos de Andrade, Diretor da \\ Eccen, Excelência em Tecnologia, que representa no Brasil a Bio-Rad Informatics Division.
}

Quanto mais complexa for uma substância, mais informações são necessárias para a sua caracterização, de modo que o emprego de múltiplas técnicas de analise para sua correta descrição é mais comum do que se pode imaginar. Este é o caso dos materiais poliméricos.

A análise e a caracterização de polímeros, incluindo os ensaios para o acompanhamento dos seus processos de degradação, podem envolver desde procedimentos e testes relativamente simples, como medidas de solubilidade e de densidade, a observação do comportamento da amostra submetida a aquecimento e testes químicos de identificação, até o uso de técnicas instrumentais sofisticadas, como as microscopias óptica e eletrônica, a difração de raios X, as análises térmicas e as espectroscopias Vibracional (IR, NIR e Raman), RNM e UV-Vis ${ }^{[1-4]}$. Além disso, os materiais poliméricos podem requerer ainda o emprego de outros métodos de análise para a determinação das suas estruturas em escala coloidal, como a cromatografia por permeação em gel $^{[5]}$ e o espalhamento de raios X em ângulos pequenos (do inglês, Small Angle X ray Scattering - SAXS ${ }^{[6]}$.

A grande maioria das análises de polímeros envolve a obtenção de espectros vibracionais, com atribuições de bandas feitas por comparação dos resultados com bases de dados especializadas, sendo a espectroscopia IR (em especial a FT-IR) uma das mais utilizadas para estes fins. Mais recentemente foi introduzida a técnica ATR-FTIR (ATR, do inglês, Attenuated Total Reflectance), igualmente muito útil para a análise de materiais poliméricos. Por ser uma técnica pontual, resolve em parte os problemas referentes à preparação da amostra e a reprodutibilidade espectral. O sistema ATR opera medindo as variações que ocorrem em um feixe de radiação IR que é totalmente refletido internamente quando em contato com a amostra ${ }^{[7]}$.

A espectroscopia Raman também tem sido empregada para este fim porque pode captar informações não disponíveis apenas pelo uso do IR. Por exemplo, esta técnica é uma ferramenta útil na obtenção de informações importantes a respeito dos mecanismos de polimerização, permitindo a otimização dos parâmetros de fabricação ${ }^{[8]}$. Pode também ser interessante em estudos sobre vulcanização (as bandas Raman de espécies de enxofre são mais ativas) e em estudos de polimerização em emulsões, porque esta técnica permite análises envolvendo soluções aquosas ${ }^{[9]}$.

O fato é que a interpretação de espectros de polímeros, bem como a identificação e a classificação de polímeros comerciais, são desafios a serem vencidos, sendo a peça chave na busca da solução de tais problemas a correlação entre espectros e estruturas, informação geralmente indisponível com a simples busca e análise espectral em coletâneas de dados publicadas em papel.

Atualmente pode-se contar como os aplicativos do KnowItAll ${ }^{\circledR}$ Informatics System da Bio-Rad Informatics Division que, associados às suas bases de dados espectrais digitais, permitem correlações muito mais precisas em um tempo de análise muito menor. Dentre os inúmeros outros campos de aplicação, pode-se agora utilizar este procedimento na interpretação espectral de materiais poliméricos, na identificação de espectros de amostras poliméricas desconhecidas, na classificação e/ou comparação de suas características e na busca e interpretação de espectros usando as bases de dados espectrais de referência, em conjunto com múltiplas técnicas de análises complementares ${ }^{[10]}$. Estes aplicativos, disponíveis para Empresas e Centros de Pesquisa, são:

\section{Analyzelt ${ }^{\mathrm{TM}}$ Polymer IR}

Desenvolvido especificamente para compostos poliméricos, ajuda na interpretação e atribuição de bandas de um espectro infravermelho (IR) de polímeros. Ele associa os intervalos de frequiência de cerca de 100 grupos característicos e suas intensidades, com gráficos de barras e os fragmentos estruturais dos grupos funcionais de compostos poliméricos, tornando-se uma peça chave na correlação espectro-estrutura. Simplesmente abra um espectro IR e clique em um pico de interesse para comparação com as bases AnalyzeIt ${ }^{\mathrm{TM}}$ Polymer IR, que gera uma lista de todos os grupos funcionais possíveis naquela posição. Pode-se também visualizar cada fragmento estrutural, juntamente com outras regiões nas quais estes fragmentos conteriam picos.

\section{HaveltAII ${ }^{\circledR}$ R}

Juntamente com as suas bases de dados espectrais SADTLER, a Bio-Rad Informatics Division oferece o acesso on-line a mais de 220.000 espectros IR de alta qualidade, totalmente integrado ao ambiente para espectroscopia KnowItAll $^{\circledR}$ Informatics System e softwares associados (ex.: Pirouette, da Infometrics, Inc.). Sua coleção espectral oferece acesso a espectros IR, NIR e ATR-FTIR, cobrindo uma ampla gama de compostos químicos puros e de produtos comerciais usados na Indústria e em Centros de Pesquisa em polímeros e em muitas outras áreas de aplicação. Esta coleção é ideal para a interpretação, verificação e classificação de espectros. Com o KnowItAll ${ }^{\circledR}$ Informatics System você poderá realizar buscas nas bases de dados HaveItAll ${ }^{\circledR}$ IR por espectros, picos, nomes, estruturas e por meio de vários outros campos, como a técnica utilizada, a massa mo- 
lar, o número de registro do Chemical Abstracts (CAS), etc, utilizando os mais poderosos algoritmos de buscas.

\section{HaveltAlI ${ }^{\circledR}$ Raman}

Este programa oferece ao usuário uma fonte confiável de dados espectrais Raman, focados em monômeros, polímeros e compostos orgânicos e inorgânicos, que podem ser usados como referência. O HaveItAll ${ }^{\circledR}$ Raman permite ao usuário importar seus próprios espectros e compará-los contra uma bases de dados contendo 4.465 espectros de alta qualidade. As buscas podem ser feitas com o KnowItAll ${ }^{\circledR}$ Informatics System por espectro, picos, nome, estrutura e subestrutura, além de vários outros campos de buscas tais como fabricante, técnica, ponto de ebulição, massa molar e o número de registro do Chemical Abstracts (CAS). Os usuários também podem acessar todas as informações disponíveis relacionadas ao espectro de referência, como fonte da amostra, solvente, condições de produção, equipamento, controle de qualidade, estudos de estabilidade, seleção de materiais, etc.

\section{HaveltAlI ${ }^{\circledR}$ NMR}

A Bio-Rad oferece também o acesso à maior coleção de dados de Ressonância Magnética Nuclear (NMR), que podem ser pesquisados com o KnowItAll ${ }^{\circledR}$ Informatics System. São, ao todo, cerca de 438.000 espectros ${ }^{13} \mathrm{C}$ NMR e 30.000 espectros ${ }^{1} \mathrm{H}$ NMR que podem ser usados como referência para previsões confiáveis. Com o HaveItAll ${ }^{\circledR} \mathrm{NMR}$, além de poder salvar os dados espectrais usados para construir as suas previsões e você poderá acessar todas as informações disponíveis a respeito do espectro de referência, tais como a fonte de obtenção da amostra, o solvente, as condições experimentais, o equipamento e as propriedades da molécula.

\section{HaveltAll ${ }^{\circledR}$ XNMR}

Usando o HaveItAll ${ }^{\circledR}$ XNMR é possível o acesso a cerca de 71.000 espectros XNMR de referência, para previsões de espectros ${ }^{19} \mathrm{~F}$ NMR, ${ }^{31} \mathrm{P}$ NMR, ${ }^{15} \mathrm{~N}$ NMR, ${ }^{11} \mathrm{~B}$ NMR, ${ }^{17} \mathrm{O}$ NMR, ${ }^{29} \mathrm{Si}$ NMR e outros núcleos, com excelente qualidade. Com o software KnowItAll PredictIt ${ }^{\mathrm{TM}} \mathrm{NMR}$, os pesquisadores poderão visualizar os dados usados para a previsão de espectros e acessar todas as informações disponíveis a respeito do espectro de referência, tais como a fonte de obtenção da amostra, o solvente, as condições experimentais, o equipamento e as propriedades da molécula.

\section{HaveltAII ${ }^{\circledR}$ MS}

A coleção de espectros do HaveItAll ${ }^{\circledR}$ MS e as demais informações associadas incluem dados do National Institute of Standards and Technology (NIST), com a assessoria de especialistas da Environmental Protection Agency (EPA) e dos National Institutes of Health (NIH). Com o KnowItAll ${ }^{\circledR}$ Informatics System você poderá realizar buscas nas bases de dados HaveItAll ${ }^{\circledR}$ MS por picos, nomes, estruturas e subestruturas, além de vários outros campos de busca, como a técnica utilizada, a massa molar, o número de registro do Chemical Abstracts (CAS). É possível ainda executar análises usando técnicas hifenadas, como o GC-MS. A base de dados também inclui sinônimos da nomenclatura química.

\section{HaveltAll ${ }^{\circledR}$ UV-Vis}

Usando o KnowItAll ${ }^{\circledR}$ Informatics System, associado às bases de dados HaveItAll ${ }^{\circledR}$ UV-Vis, tem-se acesso a espectros de alta qualidade de compostos orgânicos puros. Esta coleção é muito útil para a identificação e classificação de espectros desconhecidos. Os usuários também podem estes bancos de dados para acessar compostos usados em suas áreas de aplicação ou pesquisa, como em ciências dos materiais, dentre outras.

A licença anual do HaveItAll ${ }^{\circledR}$ oferece a possibilidade de buscas ilimitadas e acesso a todos os dados da base por um ano.

Todos estes aplicativos e bases de dados estão incluídos no programa KnowItAll ${ }^{\circledR}$ U, produzido pela Bio-Rad Informatics Division para uso exclusivo das Universidades em ensino e pesquisa acadêmica.

\section{Referências}

1. Braun, D. - Simple Methods for Identification of Plastics, Macmillan Publishing Co., Inc, 1982, 96 pp.

2. DePaoli, M-A. - Degradação e estabilização de polímeros, Editora Artliber, 2008, 286 pp.

3. Canevarolo Jr., S.V. - Técnicas de caracterização de polímeros, Editora Artliber, 2004, 446 pp.

4. Gracia, D. - Identification of Polymeric Samples FT-IR and Sadtler Database Search: An Example, Bio-Rad Technical Note (http://www.eccen.com.br/biorad/anotes).

5. Collins, C.H., Braga, G.L. \& Bonato, P. - Fundamentos de cromatografia, Editora da Unicamp, 2006, 456 pp.

6. SAXS on the Internet, em http://srs.dl.ac.uk/arch/dalai/ (acessado em 19/03/2009).

7. FT-IR Spectroscopy Attenuated Total Reflectance (ATR), Perkin Elmer Technical Note, em http://las.perkinelmer.com/content/TechnicalInfo/TCH_FTIRATR.pdf, (acessado em 20/03/2009).

8. Using Chemometrics and Raman Spectra for Quantitative Predictions of Physical and Chemical Properties of Polymers, Horiba Jobin Yvon Raman Application Note, em http://www.horiba.com/scientific/products/ raman-spectroscopy/application-notes/polymers/?L=0 (acessado em 20/03/2009).

9. Raman Characterization of Polymers in Industrial Applications, Jobin Yvon Raman Application Note, em http://www.horiba.com/scientific/products/ramanspectroscopy/application-notes/polymers/?L=0 (acessado em 20/03/2009).

10. Banik, G.M.; Scandone, M.; Tuzynski, R. e Kernan, D., A new approach to simultaneous Raman and IR spectral searches, Spectroscopy, 2005 (http://www.eccen. com.br/biorad/anotes).

Distribuidor autorizado da Bio-Rad Informatics Division no Brasil, com exclusividade para o aplicativo KnowItAll ${ }^{\circledR}$ :

ECCEN - Excelência em Tecnologia, Rua José Paulino, 2236 cj.11/23 - Vila Itapura 13.023-102 Campinas - SP Telefone: (19) 32312988 - Fax: (19) 32312914 , e-mail: diretoria@ eccen.com.br, site: www.eccen.com.br 